\title{
Relationship between Body Mass Index Reference and All-Cause Mortality: Evidence from a Large Cohort of Thai Adults
}

\author{
Vasoontara Yiengprugsawan, ${ }^{1}$ Cathy Banwell, ${ }^{1}$ Jiaying Zhao, ${ }^{1}$ \\ Sam-ang Seubsman, ${ }^{1,2}$ and Adrian C. Sleigh ${ }^{1}$ \\ ${ }^{1}$ National Centre for Epidemiology and Population Health, Research School of Population Health, College of Medicine, \\ Biology and Environment, The Australian National University, Building 62, Mills Road, Acton, Canberra, ACT 2601, Australia \\ ${ }^{2}$ School of Human Ecology, Sukhothai Thammathirat Open University, Nonthaburi 11120, Thailand \\ Correspondence should be addressed to Vasoontara Yiengprugsawan; vasoontara.yieng@anu.edu.au
}

Received 21 July 2014; Revised 24 October 2014; Accepted 25 October 2014; Published 17 November 2014

Academic Editor: Aron Weller

Copyright (c) 2014 Vasoontara Yiengprugsawan et al. This is an open access article distributed under the Creative Commons Attribution License, which permits unrestricted use, distribution, and reproduction in any medium, provided the original work is properly cited.

\begin{abstract}
We investigate variation in body mass index (BMI) reference and 5-year all-cause mortality using data from 87151 adult Open University students nationwide. Analyses focused on BMI reference bands: "normal" ( $\geq 18.5$ to $<23)$, "lower normal" ( $\geq 18.5$ to $<20.75$ ), "upper normal" ( $\geq 20.75$ to $<23$ ), and "narrow Western normal" ( $\geq 23$ to $<25$ ). We report hazard ratios (HR) and $95 \%$ Confidence Intervals adjusting for covariates. Compared to lower normal, adults aged 35-65 years who were obese (BMI $\geq 30$ ) were twice as likely to die during the follow-up (HR 2.37; 1.01-5.70). For the same group, when using narrow Western normal as the reference, the results were similar (HR 3.02; 1.26-7.22). However, different combinations of BMI exposure and reference band produce quite different results. Older age persons belonging to Asian overweight BMI category $(\geq 23$ to $<25)$ were relatively protected from mortality (HR $0.57 ; 0.34-0.96$ and HR $0.49 ; 0.28-0.84)$ when assessed using normal $(\geq 18.5$ to $<23)$ and upper normal $(\geq 20.75$ to $<23$ ) as reference bands. Use of different "normal" reference produced varying mortality relationships in a large cohort of Thai adults. Caution is needed when interpreting BMI-mortality data.
\end{abstract}

\section{Introduction}

Body mass index (BMI) in $\mathrm{kg} / \mathrm{m}^{2}$ has been commonly used in epidemiological studies as a predictor of morbidity and mortality $[1,2]$. However, consideration of differing normal reference standards for BMI when relating body size and mortality has revealed that a broader reference band can lead to artefactual evidence that overweight protects against mortality [3].

Internationally, various studies have analysed all-cause mortality against normal reference BMI cut-offs, summarized by a systematic review of 97 articles with a majority of studies using a broad "normal" category of BMI 18.5-25 [4]. However, recent research on Western populations has used a narrower BMI reference band of 22.5 to $<25$ to represent the lowest mortality group. This information is captured in a pooled analysis of 19 prospective studies of 1.46 million white adults [5] and in a collaborative study of close to 900,000 participants from Western Europe and North America [2].

We note from previous research that Caucasians and Asians differ with regard to BMI and body composition $[6,7]$ and there are also differences with regard to BMI and morbidity among different Asian groups $[8,9]$. Indeed, a report about a cohort of one million East and South Asians recently used a narrower BMI normal reference of 22.6 to $<25.0[10,11]$. Aware of these developments regarding BMI reference bands, we report whether variation in BMI reference bands produces a substantially different relationship with mortality, using our cohort of Thai adults and five-year mortality follow-up.

\section{Methods and Procedures}

This analysis is part of the overarching Thai Cohort Study, an ongoing epidemiological investigation of changing patterns 
TABLE 1: Body mass index and all-cause mortality, Thai Cohort Study (2005-2010).

\begin{tabular}{|c|c|c|c|c|c|c|}
\hline \multirow{3}{*}{ Body mass index categories } & \multicolumn{3}{|c|}{ Proportion \% } & \multicolumn{3}{|c|}{5 -year crude death rate per $100,000(n)^{*}$} \\
\hline & \multirow{2}{*}{ Overall } & \multicolumn{2}{|c|}{ Age groups } & \multirow{2}{*}{ Overall } & \multicolumn{2}{|c|}{ Age groups } \\
\hline & & $<35$ years & $35-65$ years & & $<35$ years & $35-65$ years \\
\hline Underweight $(<18.5)$ & 14.7 & 18.7 & 4.5 & $570.0(72)$ & $511.2(59)$ & $104.0(12)$ \\
\hline Lower normal $(\geq 18.5$ to $<20.75)$ & 29.1 & 33.6 & 17.7 & $488.0(122)$ & $424.3(88)$ & $154.3(32)$ \\
\hline Upper normal $(\geq 20.75$ to $<23)$ & 25.3 & 24.7 & 26.9 & $714.1(155)$ & $505.4(77)$ & $485.8(74)$ \\
\hline Overweight $(\geq 23$ to $<25)$ & 15.1 & 11.7 & 23.7 & $725.9(94)$ & $634.7(46)$ & $662.3(48)$ \\
\hline Obese I $(\geq 25$ to $<30)$ & 13.2 & 9.2 & 23.4 & $850.8(96)$ & $653.8(37)$ & $653.8(59)$ \\
\hline Obese II $(\geq 30)$ & 2.6 & 2.1 & 3.9 & $1075.3(24)$ & $463.7(6)$ & 1919.0 (18) \\
\hline
\end{tabular}

${ }^{*} n$ : actual number of deaths; total deaths $=563$ for the period $2005-2010$.

of health risks and outcomes. Data derive from a research cohort of distance-learning adult students at Sukhothai Thammathirat Open University (STOU), who resided all over Thailand. The baseline comprehensive 20-page mailout health questionnaire was sent to all STOU students enrolled in 2005 noting that participation was voluntary and 87151 responded. The cohort participants share similar certain sociogeographic attributes with the general Thai population [12]. The cohort range from 15 to 87 years at baseline in 2005 (mean age was 29 years), slightly more than half were females, and half resided in urban areas.

The baseline questionnaire collected data on a wide range of topics including sociodemogeographic information, social and environmental context, and health behaviors and states. Weight and height were also recorded. Validity of selfreported weight and height has previously been tested among 741 STOU students and showed strong correlations between self-reported and measured weight and height (Spearman's correlation for men and women, resp., 0.95 and 0.97 for weight and 0.94 and 0.94 for height) [13].

Using self-reported weight and height, we then categorised individuals using body mass index (BMI) cut-offs based on the International Obesity Task Force categories for Asians [14]: underweight (BMI < 18.5), normal $(18.5 \geq \mathrm{BMI}<$ $23)$, overweight at risk $(23 \geq \mathrm{BMI}<25)$, obese I $(25 \geq \mathrm{BMI}$ $<30$ ), and obese II (BMI $\geq 30)$. To calculate the mortality hazard ratio for a given BMI category, we used (separately) four reference BMI bands: "lower normal" $(\geq 18.5$ to $<20.75)$, "upper normal" ( $\geq 20.75$ to $<23$ ), "normal Asian" ( $\geq 18.5$ to $<23)$, "normal Western" $(\geq 23$ to $<25)$. We stratified the cohort into two age groups ( $<35$ years and $35-64$ years) and adjusted the hazard ratio for covariates.

For mortality data linkage, all cohort members have provided their Citizen ID number which was matched with death records from the Thai Ministry of Interior and subsequently linked with causes of death from the Ministry of Public Health. Up until March 2010, there were a total of 583 deaths among the Thai Cohort Study participants.

The covariates chosen were based on our previous reports for cohort analysis of body mass index [15]. These covariates included sociodemographic variables (age in years, sex, marital status, personal household monthly income categories, paid work hours, and urban-rural residence). Also, covariates included health states, self-assessed health at baseline, and health-risk behaviours (smoking and alcohol drinking). Analyses excluded cohort members who reported chronic metabolic or cardiovascular disorders including diabetes and hypertension at baseline (12.6\% of cohort members).

\section{Results}

We report BMI category prevalence overall and by two broad age groups ( $<35$ years and 35-65 years, Table 1$)$. We found that underweight was relatively much more common among the younger group (18.7\% versus $4.5 \%$ ) while overweight and obesity were much more prevalent among the older group (11.7\% versus $23.7 \%$ for overweight, $9.2 \%$ versus $23.4 \%$ for obese I category, and $2.1 \%$ versus 3.9\% for obese II category). We also present the 5-year crude all-cause mortality rate which was highest for upper obese category 1 among the younger group (653.8 per 100,000 persons) and for obese category II in the older group (1919.0 per 100,000 persons). We note also that for obese II category there were relatively few deaths (6 for the younger group and 18 for the older group) making mortality estimates for this extreme BMI category less precise than corresponding estimates for all other BMI categories. Cohort attributes were described by proportion overall and by body mass index categories (Table 2). Compared to females, males were much more likely to be overweight (21.7\% versus $9.7 \%)$ and obese I (19.7\% versus $7.8 \%$ ). Also, cohort members with higher monthly income were more likely to have higher BMI (11.3\% versus $16.8 \%$ versus $23.4 \%$ for overweight and $9.0 \%$ versus $14.8 \%$ versus $24.0 \%$ for obese I category).

Using hazard ratios and controlling for potential covariates, we tested four "normal" BMI reference bands (Table 3 ). Compared to Asian lower normal as BMI reference, cohort members aged $35-65$ years who were obese $(B M I \geq 30)$ were twice as likely to die during the five-year follow-up (HR 2.37, 95\% CI 1.01-5.70). For the same group (BMI $\geq 30$; age $\geq 35$ years), when using narrow Western normal as the reference, the results were similar (HR 3.02, 95\% CI 1.26-7.22) and indicated that obesity was a risk for mortality. However, some different combinations of BMI exposure and reference band produce quite different results. For example, older age persons belonging to Asian overweight BMI category ( $\geq 23$ to $<25$ ) were relatively protected from mortality (HR $0.57 ; 0.34-$ 0.96 and HR $0.49 ; 0.28-0.84$ ) when assessed using normal 
TABLE 2: Cohort attributes by body mass index categories, Thai Cohort Study (2005-2010).

\begin{tabular}{|c|c|c|c|c|c|c|c|}
\hline \multirow{2}{*}{ Cohort attributes } & \multirow{2}{*}{ Proportion (row \%) } & \multicolumn{6}{|c|}{ Body mass index categories (column \%) } \\
\hline & & Underweight & Lower normal & Upper normal & Overweight & Obese I & Obese II \\
\hline \multicolumn{8}{|l|}{ Sociodemographic } \\
\hline Males & 45.3 & 6.1 & 20.9 & 28.6 & 21.7 & 19.7 & 3.0 \\
\hline Females & 54.7 & 21.8 & 35.9 & 22.6 & 9.7 & 7.8 & 2.2 \\
\hline \multicolumn{8}{|l|}{ Monthly income (Baht) } \\
\hline$\leq 3000 \mathrm{Baht}$ & 10.1 & 21.0 & 33.1 & 23.6 & 11.2 & 8.3 & 2.8 \\
\hline $3001-7000$ & 30.9 & 19.4 & 33.9 & 24.4 & 11.3 & 9.0 & 2.1 \\
\hline $7001-20000$ & 47.5 & 11.9 & 27.4 & 26.5 & 16.8 & 14.8 & 2.6 \\
\hline$>20000$ & 10.6 & 6.4 & 17.6 & 24.5 & 23.4 & 24.0 & 4.1 \\
\hline \multicolumn{8}{|l|}{ Paid work } \\
\hline$\leq 10$ hours & 24.3 & 14.3 & 28.8 & 25.1 & 15.5 & 13.6 & 2.7 \\
\hline 10-40 hours & 28.2 & 11.6 & 26.9 & 25.9 & 17.0 & 15.7 & 2.8 \\
\hline$>40$ hours & 35.0 & 15.1 & 30.1 & 25.7 & 14.5 & 12.3 & 2.2 \\
\hline \multicolumn{8}{|l|}{ Residence } \\
\hline Rural & 48.2 & 14.9 & 30.0 & 25.7 & 14.9 & 12.3 & 2.2 \\
\hline Urban & 52.8 & 14.6 & 28.3 & 24.9 & 15.3 & 14.0 & 2.9 \\
\hline \multicolumn{8}{|l|}{ Health behaviours } \\
\hline \multicolumn{8}{|l|}{ Smoking } \\
\hline Never & 72.3 & 17.9 & 32.4 & 24.6 & 12.7 & 10.1 & 2.3 \\
\hline Former & 17.4 & 6.2 & 19.5 & 27.0 & 22.1 & 21.9 & 3.4 \\
\hline Current & 10.3 & 6.9 & 22.1 & 27.1 & 20.4 & 20.3 & 3.3 \\
\hline \multicolumn{8}{|l|}{ Alcohol drinking } \\
\hline Never & 26.4 & 20.3 & 33.2 & 23.2 & 11.3 & 9.6 & 2.5 \\
\hline Occasional & 59.8 & 13.3 & 28.7 & 26.1 & 15.8 & 13.5 & 2.5 \\
\hline Regular & 4.9 & 4.9 & 16.2 & 25.8 & 24.5 & 25.0 & 3.6 \\
\hline Former & 9.0 & 13.0 & 26.7 & 25.2 & 16.5 & 15.2 & 3.3 \\
\hline \multicolumn{8}{|l|}{ Physical activity } \\
\hline 0 times per week & 34.7 & 19.1 & 31.5 & 22.4 & 12.3 & 11.7 & 3.0 \\
\hline 1-3 times per week & 26.9 & 15.8 & 29.9 & 24.5 & 14.3 & 13.1 & 2.5 \\
\hline $3+$ times per week & 38.4 & 10.3 & 26.9 & 28.4 & 17.9 & 14.2 & 2.3 \\
\hline \multicolumn{8}{|l|}{ Self-assessed health } \\
\hline Excellent/good & 70.3 & 13.7 & 29.1 & 26.2 & 15.8 & 12.9 & 2.2 \\
\hline Fair & 25.1 & 16.8 & 29.2 & 23.2 & 13.7 & 13.7 & 3.4 \\
\hline Poor/very poor & 4.6 & 19.1 & 29.1 & 22.8 & 11.8 & 13.1 & 4.1 \\
\hline
\end{tabular}

$(\geq 18.5$ to $<23)$ and upper normal $(\geq 20.75$ to $<23)$ as reference bands.

\section{Discussion}

Our findings support previous methodological research showing that BMI-mortality relationships differ substantially according to the BMI band used as normal reference [3]. In our Thai data, using an Asian lower cut-off and narrow Western normal band revealed that obese II category (BMI $\geq 30$ ) was a predictor of mortality among older Thai adults. Reference of Asian normal and upper normal led to the conclusion that being overweight by Asian standard $(23 \geq$ $\mathrm{BMI}<25$ ) was strongly protective against mortality. Repeated analyses excluding the 204 injury deaths revealed little change in the results.
Our results were in accordance with Western studies $[2,5]$ and some East and South Asian studies whereby a BMI reference band of 22.5 to $<25$ was used as lowest mortality reference in these populations [10]. Our results were also similar to a Singapore Chinese Health Study which noted that BMI between 18.5 and 21.4 had the least mortality risk for those aged below 65 years [16]. Other large Asian BMI studies have investigated morbidity outcomes using the Asian BMI "normal" reference band $(18.5 \geq \mathrm{BMI}<23)$, but patterns of association were different to those for mortality as shown in our study $[14,17,18]$. Results presented here add to limited evidence on BMI and mortality in Asian populations $[19,20]$.

Our study is strengthened by the participation of a large number of Thai adults living nationwide and, in particular, by the wide array of baseline personal and health information which we used to adjust for potential confounders, including smoking status and socioeconomic status. By modest median 


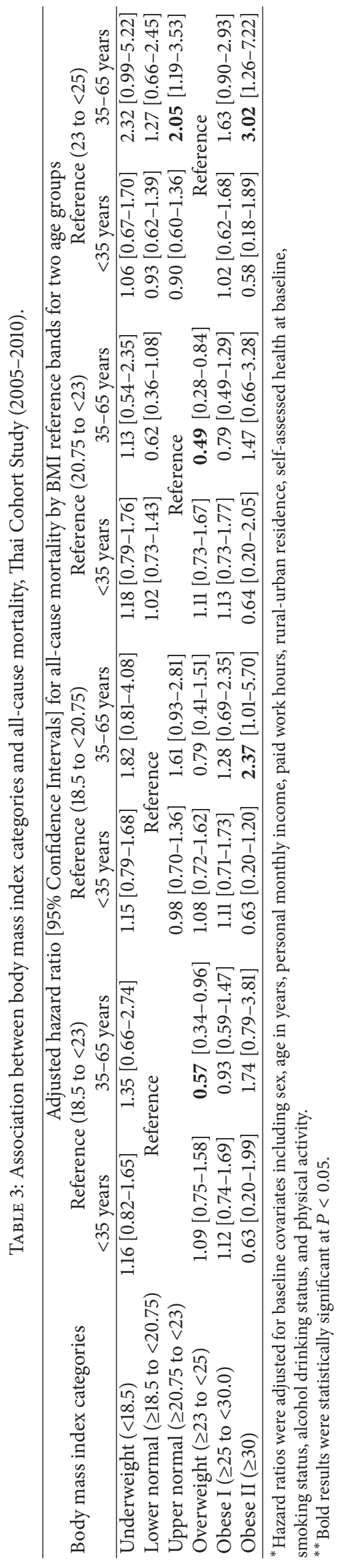


income, geographic residence, and sex ratio our cohort represented well the Thai population. Cohort members were better educated than average for Thailand but this was advantageous for collecting accurate self-reported demographic, health, and behavioural data used in the covariate-adjusted analyses presented here.

We note one of the limitations of the study was the relatively short period of follow-up resulting in small number of deaths for analyses. It is also possible that some deaths might be due to preexisting health conditions within the 5 years of initial follow-up. Given that the cohort members provided baseline information on doctor-diagnosed chronic conditions (cardiometabolic disorders) which could be intermediate variables in the BMI-mortality causal pathway, we have excluded these cohort members from the analyses. Future longitudinal analyses will have longer follow-up periods as the cohort ages and will refine the evidence on the BMI-mortality relationship.

\section{Conclusion}

Use of different BMI bands as "normal" reference categories could lead to different conclusions when analysing body size as a determinant of all-cause mortality. Our study has demonstrated the impacts of changing the normal reference cut-offs on mortality estimates among Thai adults. Caution is needed when interpreting BMI-mortality data. There is no consensus on the optimal reference band for various populations when analysing BMI-associated morbidity and mortality and this needs to be resolved.

\section{Ethical Approval}

Informed written consent was obtained from all participants. All students were advised that they could withdraw or not participate, without any effect on their academic progress. They were assured that their information was confidential and their identity would never be revealed. The questionnaires never sought sensitive personal information and no biological samples were taken. Ethics approval was obtained from Sukhothai Thammathirat Open University Research and Development Institute (Protocol 0522/10) and the Australian National University Human Research Ethics Committee (Protocols 2004/344 and 2009/570).

\section{Conflict of Interests}

The authors declare that there is no conflict of interests regarding the publication of this paper.

\section{Authors' Contribution}

Vasoontara Yiengprugsawan conceptualised, analysed, and drafted the paper with inputs from Adrian C. Sleigh, Cathy Banwell, and Jiaying Zhao. Adrian C. Sleigh and Sam-ang Seubsman led the Thai Cohort Study.

\section{Acknowledgments}

This study was supported by the International Collaborative Research Grants Scheme with joint grants from the Wellcome Trust UK (GR071587MA) and the Australian National Health and Medical Research Council (268055) and as a global health grant from the NHMRC (585426). The authors thank the staff at Sukhothai Thammathirat Open University (STOU) who assisted with student contact and the STOU students who are participating in the cohort study. They also thank Dr. Bandit Thinkamrop and his team from Khon Kaen University for support on initial data processing. Also they would like to thank the Thai Ministry of Interior and Ministry of Public Health for mortality investigation and cause of death data linkage.

\section{References}

[1] H.-L. Wee, Y. Wu, J. Thumboo, J. Lee, and E. S. Tai, "Association of body mass index with Short-Form 36 physical and mental component summary scores in a multiethnic Asian population," International Journal of Obesity, vol. 34, no. 6, pp. 1034-1043, 2010.

[2] Prospective Studies Collaboration, G. Whitlock, S. Lewington, P. Sherliker et al., "Body-mass index and cause-specific mortality in 900000 adults: collaborative analyses of 57 prospective studies," The Lancet, vol. 373, no. 9669, pp. 1083-1096, 2009.

[3] E. Banks, G. Joshy, and R. Korda, "Monday's medical myth: overweight people live longer," The Conversation, http://theconversation.com/mondays-medical-myth-overweight-people-livelonger-12825.

[4] K. M. Flegal, B. K. Kit, H. Orpana, and B. I. Graubard, "Association of all-cause mortality with overweight and obesity using standard body mass index categories a systematic review and meta-analysis," The Journal of the American Medical Association, vol. 309, no. 1, pp. 71-82, 2013.

[5] A. B. de Gonzalez, P. Hartge, J. R. Cerhan et al., "Body-mass index and mortality among 1.46 million white adults," The New England Journal of Medicine, vol. 363, no. 23, pp. 2211-2219, 2010.

[6] P. Deurenberg, M. Deurenberg-Yap, and S. Guricci, "Asians are different from Caucasians and from each other in their body mass index/body fat per cent relationship," Obesity Reviews, vol. 3, no. 3, pp. 141-146, 2002.

[7] R. C. Weisell, "Body mass index as an indicator of obesity", Asia Pacific Journal of Clinical Nutrition, vol. 11, supplement 8, pp. S681-S684, 2002.

[8] N. T. Tuan, L. S. Adair, C. M. Suchindran, H. Ka, and B. M. Popkin, "The association between body mass index and hypertension is different between East and Southeast Asians," The American Journal of Clinical Nutrition, vol. 89, no. 6, pp. 19051912, 2009.

[9] Y. Chen, W. K. Copeland, R. Vedanthan et al., "Association between body mass index and cardiovascular disease mortality in East Asians and South Asians: pooled analysis of prospective data from the Asia Cohort Consortium," British Medical Journal, vol. 347, article f5446, 2013.

[10] W. Zheng, D. F. McLerran, B. Rolland et al., "Association between body-mass index and risk of death in more than 1 million Asians," The New England Journal of Medicine, vol. 364, no. 8, pp. 719-729, 2011. 
[11] WHO Expert Consultation, "Appropriate body-mass index for Asian populations and its implications for policy and intervention strategies," The Lancet, vol. 364, no. 9403, pp. 157-163, 2004.

[12] A. C. Sleigh, S.-A. Seubsman, C. Bain et al., "Cohort profile: the Thai cohort of 87134 open university students," International Journal of Epidemiology, vol. 37, no. 2, pp. 266-272, 2008.

[13] L. L. Y. Lim, S.-A. Seubsman, and A. Sleigh, "Validity of selfreported weight, height, and body mass index among university students in Thailand: implications for population studies of obesity in developing countries," Population Health Metrics, vol. 7, article 15, 2009.

[14] M. Kanazawa, N. Yoshiike, T. Osaka, Y. Numba, P. Zimmet, and S. Inoue, "Criteria and classification of obesity in Japan and Asia-Oceania," Asia Pacific Journal of Clinical Nutrition, vol. 11, supplement 8, pp. S732-S737, 2002.

[15] C. Banwell, L. Lim, S. A. Seubsman, C. Bain, J. Dixon, and A. Sleigh, "Body mass index and health-related behaviours in a national cohort of 87134 Thai open university students," Journal of Epidemiology \& Community Health, vol. 63, no. 5, pp. 366372, 2009.

[16] A. O. Odegaard, M. A. Pereira, W.-P. Koh et al., "BMI, all-cause and cause-specific mortality in Chinese Singaporean men and women: the Singapore Chinese health study," PLoS ONE, vol. 5, no. 11, Article ID e14000, 2010.

[17] C. N. Mhurchu, V. Parag, M. Nakamura, A. Patel, A. Rodgers, and T. H. Lam, "Body mass index and risk of diabetes mellitus in the Asia-Pacific region," Asia Pacific Journal of Clinical Nutrition, vol. 15, no. 2, pp. 127-133, 2006.

[18] P. Boffetta, D. McLerran, Y. Chen et al., "Body mass index and diabetes in Asia: a cross-sectional pooled analysis of 900,000 individuals in the Asia cohort consortium," PLOS ONE, vol. 6, no. 6, Article ID e19930, 2011.

[19] J. Stevens and E. M. Nowicki, "Body mass index and mortality in Asian populations: implications for obesity cut-points," Nutrition Reviews, vol. 61, no. 3, pp. 104-107, 2003.

[20] S. Woo Oh, S.-A. Shin, Y. Ho Yun, T. Yoo, and B.-Y. Huh, "Cut-off point of BMI and obesity-related comorbidities and mortality in middle-aged Koreans," Obesity Research, vol. 12, no. 12, pp. 2031-2040, 2004. 


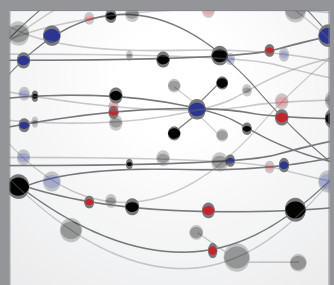

The Scientific World Journal
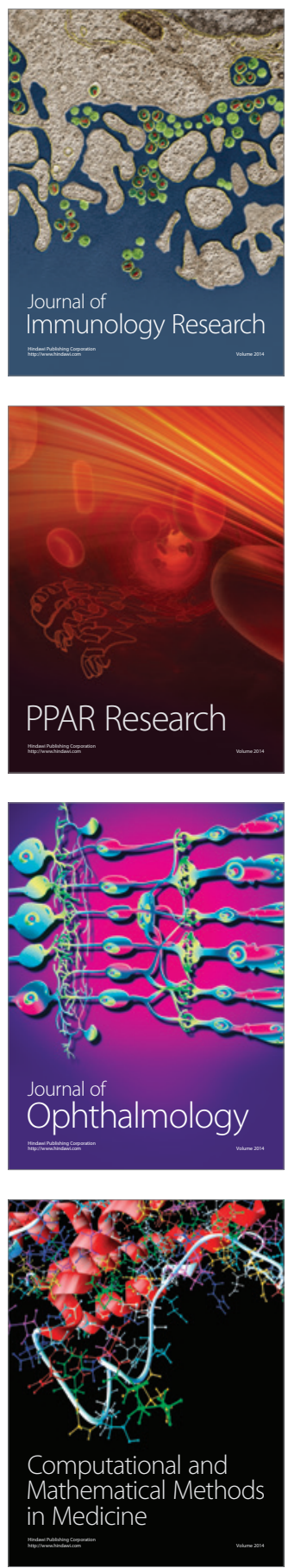

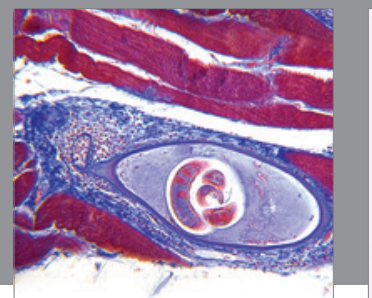

Gastroenterology

Research and Practice
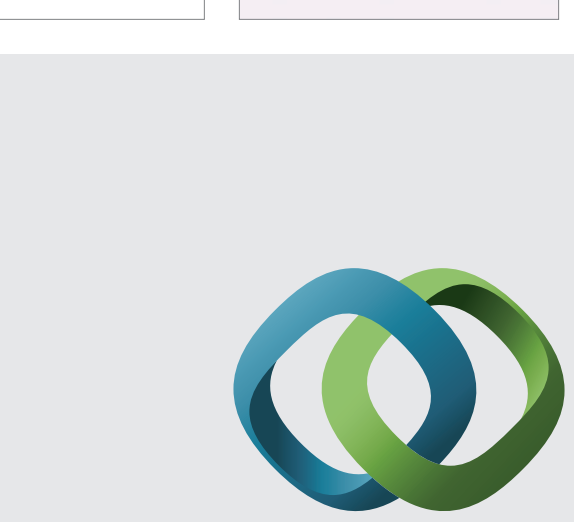

\section{Hindawi}

Submit your manuscripts at

http://www.hindawi.com
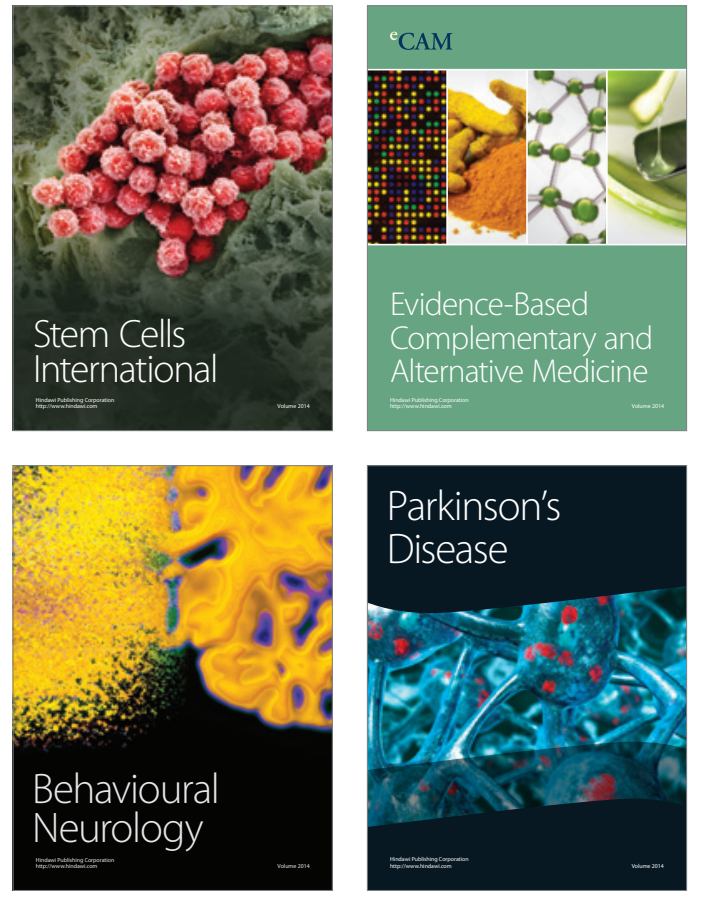
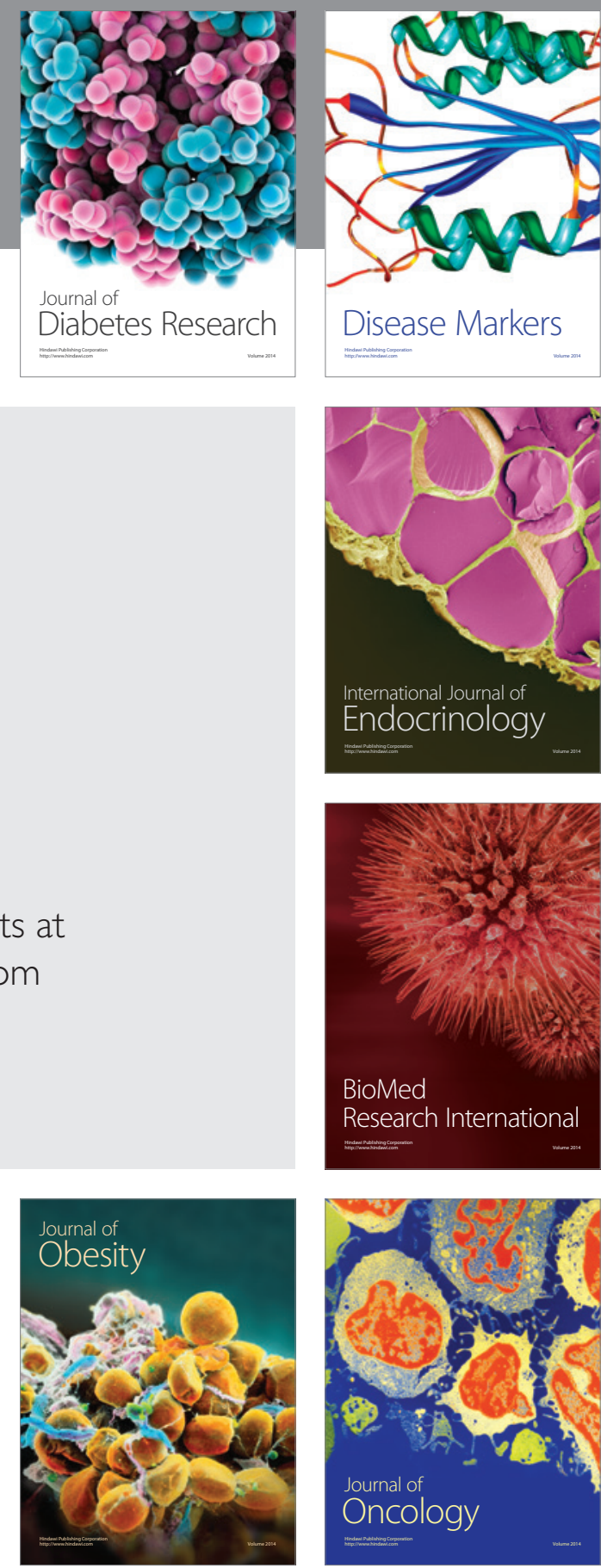

Disease Markers
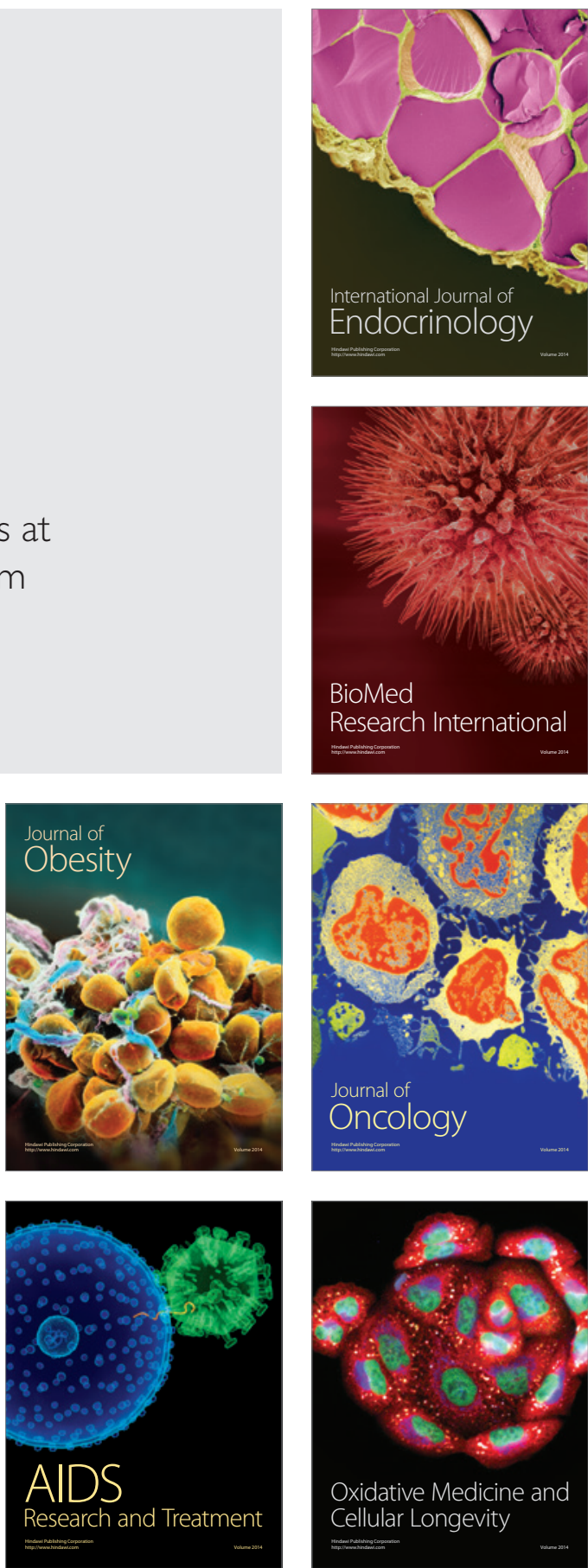